\title{
Problemas de la articulación entre fenomenología y hermenéutica en la obra de J.-L. Marion, en diálogo con la propuesta de C. Romano
}

\author{
Jorge Luis Roggero ${ }^{1}$
}

Recibido: 19 de junio de 2019 / Aceptado: 5 de abril de 2020

Resumen. Este artículo se propone examinar el modo en que la fenomenología de la donación puede lograr una vinculación exitosa con la hermenéutica. Siguiendo la indicación dada por el propio Marion, a través de una comparación de su planteo con el de Claude Romano, es posible establecer hasta qué punto la propuesta marioniana es capaz de responder a las exigencias hermenéuticas y fenomenológicas que la articulación demanda.

Palabras clave: fenomenología; hermenéutica; Jean-Luc Marion; Claude Romano; lo antepredicativo; Sprachlichkeit; esencia; sentido.

\section{[en] Problems of the Articulation between Phenomenology and Hermeneutics in J.-L. Marion's work, in dialogue with C. Romano's proposal}

\begin{abstract}
This article aims to examine the way in which the phenomenology of donation can achieve a successful articulation with hermeneutics. Following the indication given by Marion himself, through a comparison of his approach with that of Claude Romano, it is possible to establish to what extent the Marionian proposal is capable of responding to the hermeneutical and phenomenological demands that the articulation requires.

Keywords: phenomenology; hermeneutics; Jean-Luc Marion; Claude Romano; the antepredicative; Sprachlichkeit; essence; meaning.

Sumario: 1. La "tesis fenomenológica"; 2. La "exigencia fenomenológica" y la "exigencia hermenéutica"; 3. Gadamer y/o Ricœur; 4. Las tensiones entre fenomenología y hermenéutica en Sein und Zeit; 5. Reglas de la experiencia y verdades de esencia; 6. La fenomenología hermenéutica marioniana; 7. Referencias bibliográficas.
\end{abstract}

Cómo citar: Roggero, J. L. (2020) "Problemas de la articulación entre fenomenología y hermenéutica en la obra de J.-L. Marion, en diálogo con la propuesta de C. Romano", en Logos. Anales del Seminario de Metafisica 53, 327-343.

\footnotetext{
1 Centro de Estudios Filosóficos, Academia Nacional de Ciencias de Buenos Aires

Facultad de Filosofía y Letras, Universidad de Buenos Aires

Consejo Nacional de Investigaciones Científicas y Técnicas

jorgeluisroggero@gmail.com
} 
La fenomenología de la donación de Jean-Luc Marion ha recibido una objeción sostenida de modo persistente por diversos comentaristas desde la publicación de Réduction et donation en $1989 .^{2}$ Esta crítica consiste, en sus diversas expresiones, en señalar la necesidad de aclarar la función de la hermenéutica-si es que realmente hay una- en su propuesta fenomenológica. En 2011, en ocasión del Coloquio "Nouvelles phénoménologies en France", en su conferencia: "Quelques précisions sur la réduction, le donné, l'herméneutique et la donation” (2014a), Marion respondió por primera vez, de modo explícito, a esta objeción. El texto fue ampliado como capítulo II de su libro de 2016, Reprise du donné. Según el fenomenólogo francés, la hermenéutica cumple la función clave de gestionar la distancia entre lo que se da y lo que se muestra (cf. 2016, pp. 89 y 97). Esta labor de pasaje de la donación a la fenomenalización, según Marion, ya puede encontrarse operando de cuatro modos distintos en sus obras anteriores. ${ }^{3}$ Sin embargo, el carácter aun escueto de su respuesta trae como consecuencia que algunas problemáticas de la articulación entre la fenomenología y la hermenéutica se encuentren insuficientemente tematizadas por el autor. Como hilo conductor para explorar algunas de las cuestiones que quedan en suspenso, propongo prestar atención a las referencias que aparecen en el capítulo de Reprise du donné. Marion nombra explícitamente a Heidegger y a Gadamer. Pero también, en una nota al pie agregada al final del capítulo, Marion hace una última referencia con la que procura sintetizar toda su propuesta: "Así nos unimos a la tesis de C. Romano: 'la hermenéutica auténtica es una fenomenología y la fenomenología solo se cumple como hermenéutica"” (2016, p. 97 n. 2). ${ }^{4}$

Teniendo en cuenta esta indicación fundamental dada por el propio Marion -por medio de un examen del detallado análisis que propone Romano de las diversas artistas de la relación entre fenomenología y hermenéutica- puede establecerse en qué medida la fenomenología de la donación logra responder a los diversos desafíos que plantea la relación entre fenomenología y hermenéutica. Nuestra hipótesis es, pues, que si se presta atención a las posibilidades de la propuesta fenomenológica marioniana, aun cuando el autor no haya dado una respuesta explícita a todas las problemáticas, es posible esbozar el modo en que su fenomenología puede articularse productivamente con una hermenéutica respetando tanto las exigencias fenomenológicas como las hermenéuticas.

Con el objeto de demostrar estos extremos, me detendré en cada una de las ideas y de los análisis propuestos por Romano, así como de sus lecturas de Heidegger, Gadamer y Ricœur, e intentaré dar cuenta de las posibilidades de la fenomenología de la donación para responder a esas cuestiones. Finalmente extraeré una conclusión general.

\section{La "tesis fenomenológica"}

El voluminoso libro de Romano, Au cour de la raison, la phénoménologie, revela de entrada cuál es su finalidad, que no es otra que esclarecer y justificar la idea

\footnotetext{
Entre otros: Greisch (1991); Grondin (1992); MacKinlay (2010); Serban (2012).

Hice una lectura de esto cuatro modos proponiendo entenderlos como cuatro niveles de hermenéutica en mi artículo "Los cuatro niveles de hermenéutica en la fenomenología de J.-L. Marion". Cf. Roggero (2020).

4 La nota se encuentra en referencia al siguiente pasaje: "La fenomenología de la donación, por tanto, solo gestiona la distancia entre lo que se da y lo que se muestra [...] por el ejercicio de una hermenéutica propiamente fenomenológica" (p. 97).
} 
misma de fenomenología. En el "Avant-propos", Romano destaca la importancia de advertir el "problema fenomenológico", que radica en determinar si efectivamente la experiencia posee estructuras inmanentes necesarias y autónomas respecto del lenguaje y los esquemas conceptuales (cf. 2010, p. 11). ${ }^{5}$ Según el autor no puede aceptarse sin más las implicancias del "giro lingüístico". La filosofía de la experiencia no devino superflua, por el contrario, es necesario que ella haga valer sus derechos. $\mathrm{Y}$, en este sentido, Romano propone acertadamente sostener la siguiente "tesis fenomenológica": "hay una autonomía del orden prelingüístico de la experiencia 'antepredicativa', como habría dicho Husserl, respecto de las formas superiores de pensamiento y el lenguaje. La experiencia posee un lógos inmanente que la fenomenología, precisamente, tiene por propósito sacar a la luz. Es más, el lenguaje mismo sólo deviene plenamente inteligible si se lo restituye a su relación con una inteligencia prelingüística" (2010, p. 12).

Esta "tesis fenomenológica" será el contenido de la "exigencia fenomenológica" que debe respetarse en la articulación con la hermenéutica. Marion es perfectamente consciente de esta cuestión, aunque difiere con el Husserl de Erfahrung und Urteil en su concepción de lo "antepredicativo", pues para la fenomenología de la donación no se trata de buscar la génesis de la lógica ni de -finalmente- reconducir lo "antepredicativo" a una esfera "teórica", sino de indagar en lo preteórico en búsqueda de un lógos no supeditable al plano teórico y válido principalmente para la práctica. ${ }^{6}$

Con el objeto de defender la "tesis fenomenológica", Romano entiende necesario emprender una crítica severa de cierto "marco kantiano" imperante en la filosofía contemporánea. Este "marco" consiste en "concebir todo orden y toda estructuración de la experiencia como extrínseca a ella, como surgida del lenguaje, de la cultura o de sus esquemas" (2010, p. 13). Romano recuerda que la fenomenología propone una noción original de experiencia que va más allá del debate entre el empirismo y el kantismo. Según Romano, el "marco kantiano" se observa en la estrategia de cierta filosofía lingüística que sitúa al lenguaje en el lugar de las categorías kantianas: gran parte de la filosofía analítica, como el neokantismo criticado por Husserl, opera con "construcciones que viene desde arriba" (2010, pp.12-13). Este "marco kantiano" -como examinaremos en breve- representará el peligro oculto en la "exigencia hermenéutica" si se la absolutiza.

Ahora bien, estos objetivos implican analizar el estatuto de la experiencia y del lenguaje, y esto conlleva repensar a la razón misma. Romano señala que la fenomenología propone una "nueva imagen de la razón". Hay un lógos de lo sensible que debe ser desentrañado. En Formale und transzendentale Logik, Husserl habla de un "lógos del mundo estético" (Logos der ästhetischen Welt) (Hua XVII, p. 297). Más allá de la lógica de la matemática y de la ciencia, hay una lógica del mundo que descansa sobre una epifanía sensible. Pero para poder hablar de un lógos

5 De algún modo, el "problema fenomenológico" enunciado por Romano retoma el problema de la "paradoja del lenguaje" enunciado por Ricœur: "Por un lado, el lenguaje no es primero, ni tampoco autónomo; es solamente la expresión de una aprehensión de la realidad, articulada por debajo de él; y, sin embargo, es siempre en el lenguaje que se deja decir su propia dependencia respecto de lo que lo precede" (1971, p. 776). Romano reformula esta idea de modo elocuente: "Las cosas significan para nosotros antes del lenguaje. ¿Qué significan? Sólo el lenguaje puede decírnoslo. Pero el lenguaje no creó esa significación” (2010, p. 629).

6 Esta idea fue sostenida por Jean-Luc Marion en una entrevista que tuvo lugar el 9 de noviembre de 2016 en su domicilio personal en Paris. Este lógos de la práctica es el lógos del amor, el lógos del pascaliano orden del corazón, que debe completar el lógos científico-objetivante del segundo orden (cf. Marion, 1986, pp. 293-378). 
inmanente a lo sensible, destaca Romano, hay que dejar de pensar la experiencia como irracional. En este sentido, ni Marion ni la fenomenología entienden que es posible reducir a la experiencia a un puro dato bruto (empirismo). Pero, por otra parte, tampoco es posible considerar que lo dado en ella debe ser completado y estructurado por conceptos (neokantismo). Husserl convalida cierta racionalidad inmanente a la experiencia. Por este motivo, en Krisis, critica la "racionalidad de corazón estrecho" (enghertzige Rationalität) (Hua VI, p. 14). Romano enfatiza que es necesario pensar una razón ampliada capaz de incluir a su otro: la sensibilidad, la experiencia, lo antepredicativo, una "razón de gran corazón" (raison au grand cour) que rehabilite el mundo sensible como necesario para su propia existencia (Romano, 2010, pp. 14-15).

¿Cuál sería la posición de Marion ante estos objetivos? Es posible afirmar que la "tesis fenomenológica" es sostenida por Marion. La distinción entre la instancia de la donación y la instancia de la fenomenalización -es decir, la tesis de Étant donné en la que se sostiene que "todo lo que se muestra se da" (1998, p. 424), aunque no todo lo que se da se muestra, pues "si todo lo que se muestra debe primero darse, sucede a veces que lo que se da no logra, sin embargo, mostrarse" (p. 425)-implica la afirmación de una instancia antepredicativa fundante, la donación (llamada), que condiciona toda posible fenomenalización en una respuesta (cf. 1998, p. 408).

Y esta tarea de gestión del pasaje entre lo que se da y lo que se muestra es precisamente la tarea asignada a la hermenéutica. Por este motivo Marion destaca que en su hermenéutica el "en tanto que" apofántico depende del "en tanto que" hermenéutico-existencial (cf. 2016, pp. 84-88). El modo de evitar la arbitrariedad y el idealismo del "marco kantiano" es, precisamente, afirmando un lógos inmanente a la donación como instancia antepredicativa originaria que determina la interpretación o, mejor dicho, explicitación (Auslegung) hermenéutica.

Por otra parte, la fenomenología de la donación asume la originalidad de la noción fenomenológica de experiencia y la radicaliza a partir de la idea de una "contraexperiencia", que se desentiende de todo a priori (horizonte y yo). ${ }^{7}$ Asimismo, también hace suya la tarea de presentar una "nueva imagen de la razón", una "razón de gran corazón" que integre también a la racionalidad lo que Marion designa nietzscheanamente como la "gran razón": el lógos del amor. ${ }^{8}$

\section{La "exigencia fenomenológica" y la "exigencia hermenéutica"}

En el capítulo XXII de su libro, Romano se detiene en la relación entre fenomenología y hermenéutica. La reflexión comienza analizando la supuesta oposición entre ambas. Por un lado, la fenomenología es caracterizada como la ciencia que busca los comienzos absolutos y las fundaciones últimas. Por otro lado, la hermenéutica se distingue de ella porque repara en la historicidad y en la finitud de la razón, en el condicionamiento histórico de la actividad filosófica y en la necesidad de interpretación. La filosofía no puede pretender un comienzo sin supuestos porque

\footnotetext{
"La contra-experiencia ofrece la experiencia de lo que contradice irreductiblemente las condiciones de la experiencia de los objetos" (Marion, 1998, p. 301).

8 Según Marion, es necesario llevar a cabo una ampliación de la razón, es necesario constituir una "gran razón" que permita incluir al pascaliano orden del corazón. Los fenómenos decisivos solo se tornan accesibles desde la mirada del amor (cf. Marion, 2010, p. 13).
} 
se encuentra siempre in medias res. Según el autor, este es el planteo por el que se introduce un "abismo" (abîme) (cf. Ricœur, 1986, p. 40) entre fenomenología y hermenéutica (cf. Romano, 2010, p. 873).

Sin embargo, Romano destaca que esta oposición es superficial por dos motivos. En primer lugar, utilizando el mismo argumento de Paul Ricœur, Romano señala que no puede equipararse sin más a toda la fenomenología con la fenomenología idealista trascendental husserliana. ${ }^{9} \mathrm{Y}$ en segundo lugar, por medio de esta contraposición se restringen también la tarea y las posibilidades de la hermenéutica (cf. Romano, 2010, p. 874). La tesis de Romano es que "la hermenéutica auténtica es una fenomenología y la fenomenología sólo se cumple como hermenéutica" (p. 874). No hay que plantear que entre ellas existe un abismo, ni tampoco que es necesario proceder a ningún "injerto" (greffe) (Ricœur, 2013, p. 23). Según Romano, hay que entender a la fenomenología y a la hermenéutica como "floraciones de un mismo brote" (2010, p. 874).

La hermenéutica -sostiene Romano- cumple la particular función de revelarle a la fenomenología dos cuestiones que ésta parece ignorar o pretender olvidar. 1) La primera es la imposibilidad de realizar la pretensión de una Vorurteilslosigkeit. La hermenéutica le recuerda a la fenomenología que no es posible liberarse de los condicionamientos históricos. Como bien destaca Heidegger, también la investigación ontológica-fenomenológica está determinada por su situación histórica (cf. GA 24, p. 31). En la fenomenología intervienen tanto esquemas conceptuales tomados de la tradición como presupuestos históricos.

2) La segunda cuestión que la hermenéutica plantea a la fenomenología es que el lenguaje tiene un carácter constitutivo para el pensamiento. Romano destaca: "Que el lenguaje sea constitutivo para el pensamiento significa no solamente que no podríamos tener la mayoría de nuestros pensamientos, creencias u opiniones si no poseyéramos el lenguaje, sino que no podríamos tampoco tener ciertos sentimientos, objetivos, intenciones, deseos, etc." (2010, p. 877).

Esta segunda cuestión tiene una importancia decisiva en la que conviene detenerse. Romano sostiene que es fundamental articular esta "exigencia hermenéutica" con una "exigencia fenomenológica". Por un lado, la hermenéutica exige aceptar que el lenguaje es constitutivo del pensamiento en el sentido de que no es posible formular una descripción fenomenológica que no consista en una operación lingüística en la que intervengan esquemas conceptuales y presupuestos heredados de la tradición. Esto obliga a admitir que el acceso a los fenómenos es siempre mediado por algún tipo de interpretación histórica (cf. 2010, p. 887). Pero, por otro lado, la fenomenología exige advertir que existe una experiencia primera del mundo que responde a un orden inmanente. Este orden contiene un sentido pre-conceptual que debe ser analizado en sus propios términos: "hay una inteligencia de lo sensible (genitivo subjetivo) que no es una forma degradada de pensamiento o de juicio: percibir, no es ni pensar, ni juzgar, ni interpretar" (Romano, 2010, pp. 886-887).

Romano destaca que el fracaso en conciliar estas dos exigencias implica el riesgo de ceder a la radicalidad de la exigencia hermenéutica y así caer en un "idealismo lingüístico" que niegue la posibilidad de una experiencia que no esté lingüísticamente estructurada, y absolutice el lenguaje al punto de afirmar que sin él no son posibles ni identidades ni diferencia ni estructuras. Para este "idealismo lingüístico" el

9 "Lo que la hermenéutica derrumbó no es la fenomenología, sino una de sus interpretaciones, la interpretación idealista hecha por Husserl” (Ricœur, 1986, p. 39). 
mundo no posee ninguna estructura de esencia más allá de las reglas que proyecta la gramática sobre él. Ahora bien, ¿la hermenéutica efectivamente adhiere a este tipo de idealismo? (cf. Romano, 2010, pp. 877-878).

\section{Gadamer y/o Ricœur}

Romano destaca que lo primero que hay que señalar respecto de este interrogante es que no existe algo así como "la" hermenéutica sin más. Al menos, antes de responder, conviene evaluar las diferencias y coincidencias entre las propuestas de los dos autores más representativos de esta corriente filosófica: Hans-Georg Gadamer y Paul Ricœur. En primer lugar, Romano se detiene en las diferencias respecto de cinco cuestiones: 1) el concepto de hermenéutica, 2) la relación entre fenomenología y hermenéutica, 3) el modelo de hermenéutica practicada, 4) la posición respecto la tensión entre comprensión y explicación, 5) la posición respecto del cogito.

1) En relación con el concepto de hermenéutica, Gadamer persigue una descripción de la comprensión y del modo en que ésta opera en todas las ciencias del espíritu. Por su parte, Ricœur -según Romano- presenta un concepto más restringido de hermenéutica, porque ésta se ciñe a poner en práctica una interpretación de símbolos que viene, en todo caso, a completar la fenomenología husserliana. Habría que agregar que esa interpretación de símbolos es la primera etapa del rodeo de la "vía larga" ricœuriana a través del diálogo con las ciencias humanas, pero que procura reconducir finalmente a una hermenéutica ontológica. ${ }^{10}$

En este primer punto, ciertamente, Marion está más cerca de Gadamer que de Ricœur. Está claro que la hermenéutica marioniana se inscribe en la "vía corta". ${ }^{11} \mathrm{Si}$ bien Marion no hace del fenómeno de la comprensión un tema de su fenomenología, su hermenéutica y su tarea de interpretación se asienta heideggerianamente en el adonado como uno de sus modos de ser y ese es su punto de partida. Puede afirmarse que hay una suerte de "Analítica del adonado" en Le phénomène erotique, aunque la pregunta sería: “¿qué es un ser cuyo ser consiste en amar (en comprender desde el amor)?". ${ }^{12}$

10 "La vía larga que propongo también tiene por ambición dirigir la reflexión al plano de una ontología; pero lo hará gradualmente, siguiendo las exigencias sucesivas de la semántica (§ 3) y, luego, de la reflexión (§ 4). La duda que planteo al final de este parágrafo se extiende solo sobre la posibilidad de hacer una ontología directa, sustraída desde el comienzo a toda exigencia metodológica y, en consecuencia, también sustraída al círculo de la interpretación, del cual ella misma hace la teoría. Sin embargo, es el deseo de esa ontología el que mueve la empresa aquí propuesta y el que le permite no empantanarse en una filosofía lingüística a la manera de Wittgenstein, ni en una filosofía reflexiva de tipo neokantiana" (Ricœur, 2013, pp. 27-28). Romano reconoce que, posteriormente, Ricœur amplía su concepción de la hermenéutica como "la teoría de las operaciones de la comprensión en su relación con la interpretación de textos” (Ricœur, 1986, p. 75), pero que nunca dejó de considerar a la hermenéutica como una teoría de las objetivaciones y de las mediaciones simbólicas (cf. Romano, 2010, p. 879).

11 "La vía corta es la de una ontología de la comprensión, a la manera de Heidegger. Llamo 'vía corta' a esta ontología de la comprensión porque, al romper con los debates de método, se inscribe de entrada en el plano de una ontología del ser finito, y reconoce en él el comprender no ya como un modo de conocimiento, sino como un modo de ser. No se ingresa de a poco en esta ontología de la comprensión; no se accede a ella gradualmente, profundizando las exigencias metodológicas de la exégesis, de la historia o del psicoanálisis: nos transportamos en ella por una súbita inversión de la problemática. La pregunta: ¿cuáles son las condiciones necesarias para que un sujeto cognoscente puede comprender un texto, o la historia misma?, se sustituye por esta otra pregunta: ¿qué es un ser cuyo ser consiste en comprender? El problema hermenéutico se convierte así en una región de la Analítica de ese ser, el Dasein, que existe al comprender" (Ricœur, 2013, pp. 26-27).

12 Agradezco a Marcos Jasminoy por haberme hecho notar que puede leerse Le phénomène erotique como una suerte de analítica existenciaria similar a la heideggeriana. 
2) Respecto de la relación entre fenomenología y hermenéutica. Romano señala que mientras Gadamer entiende que ambas se encuentran entrelazadas y confunden sus destinos, Ricœur sostiene que hay una heterogeneidad de principio entre ambas y, por este motivo, es necesario un "injerto" de la hermenéutica en la fenomenología (cf. 2010, p. 879). Nuevamente, sobre esta cuestión cabe hacer una aclaración: la posición de Ricœur se modifica en "Phénoménologie et herméneutique. En venant de Husserl". Allí, la idea de la necesidad de un "injerto" es revisada a partir de la tesis de una presuposición recíproca, similar-por cierto-a la propuesta por Romano. Dice Ricœur: "más que una simple oposición, lo que se da entre fenomenología y hermenéutica es una interdependencia que es importante explicitar. Esta dependencia puede percibirse tanto a partir de una como de otra. Por una parte, la hermenéutica se construye sobre la base de la fenomenología y así conserva aquello de lo cual no obstante se aleja. La fenomenología sigue siendo el presupuesto insuperable de la hermenéutica. Por otra parte, la fenomenología no puede constituirse a sí misma sin un presupuesto hermenéutico" (Ricœur, 1986, p. 40). ${ }^{13}$

Marion, en este punto -como ya hemos visto-, sostiene que comparte la opinión de Romano respecto de la fuerte imbricación entre ambas.

3) Respecto del "modelo de hermenéutica", Gadamer propone el diálogo como paradigma de escucha del otro. Este modelo se aplica al texto solo de modo derivado. Por su parte, destaca Romano, Ricœur rechaza el modelo dialógico y enfatiza la diferencia entre el texto y la conversación: "lo escrito se aparta de los límites del diálogo" (1986, p. 31).

Con relación a esta cuestión, cabe destacar que Marion sigue el modelo gadameriano de la pregunta y la respuesta, pero entendido bajo la modalidad de la llamada y la respuesta.

4) Respecto del vínculo entre comprensión y explicación, Romano destaca que Gadamer -siguiendo a Dilthey y la hermenéutica romántica, pero también a Heidegger- asume una dicotomía fuerte entre ambas. Por su parte, Ricœur propone con su "vía larga" una integración de la explicación en los procesos hermenéuticos, como uno de sus momentos (cf. Romano, 2010, pp. 879-880). Ricœur entiende que hay que pensar el vínculo entre explicación y comprensión de modo dialéctico (cf. Ricœur, 1986, p. 33). Marion no se pronuncia sobre esta cuestión, pero cabe presuponer que su hermenéutica de "vía corta" se desentiende de la explicación.

5) Finalmente, respecto del cogito también son diversas las posiciones de estos dos autores. Gadamer insiste en la necesidad de deconstruir el cogito y la filosofía del sujeto. Por el contrario, Ricœur propone una filosofía reflexiva post-kantiana (siguiendo a Nabert) que no recusa la fenomenología de la conciencia, sino solamente su versión idealista.

Marion, en esta cuestión, es ciertamente más cercano a Gadamer al proponer al adonado como "lo que viene después del sujeto", pero su fenomenología en tanto crítica al idealismo husserliano, a pesar de las ostensibles diferencias con el proyecto ricœuriano, comparte algunas de las objeciones presentes en "Phénoménologie et herméneutique. En venant de Husserl”. En primer lugar, Marion coincide en la crítica al supuesto husserliano de que la subjetividad sea el lugar de la fundamentación última. Y si bien Marion no desarrolla una teoría del texto, sí concuerda con Ricœur

13 Este desplazamiento (del "injerto" a la presuposición recíproca) en el pensamiento de Ricœur ha sido señalado por Marc-Antoine Vallée (cf. 2012, p. 28 n. 25). 
en considerar que la comprensión no surge en primera instancia como un acto de la subjetividad, sino solo en segundo lugar, como "respuesta" a la cosa del texto, a las proposiciones de sentido presentes en él; aunque cronológicamente puedan darse en simultáneo (cf. Ricœur, 1986, pp. 49-55).

Luego de exponer estas diferencias entre Gadamer y Ricœur, Romano destaca que ambos proyectos comparten un supuesto fundamental: coinciden en afirmar la Sprachlichkeit, el carácter lingüístico de la experiencia del mundo. Pero ¿cómo hay que entender esta idea? Romano se detiene primero en el caso de Gadamer. Cabe hacer un análisis de la Sprachlichkeit a partir de su famoso dictum: Sein, dass verstanden werden kann, ist Sprache ("el ser que puede ser comprendido es lenguaje") (Gadamer, 1999, p. 478). Romano sostiene que esta frase lleva a afirmar que toda comprensión será un proceso lingüístico, aun cuando se trate de lo extralingüístico, pues "la idealización del lenguaje [...] habita ya toda adquisición de experiencia” (Gadamer, 1999, p. 353). En este sentido, la afirmación del hermeneuta alemán parece adherir al "idealismo lingüístico". Gadamer entiende que el mundo solo es mundo por intermedio del lenguaje y somos-en-el-mundo porque tenemos lenguaje (cf. Gadamer, 1999, p. 447 y Romano, 2010, pp. 881-883).

Romano señala que Ricœur sigue a Gadamer en esta interpretación de la Sprachlichkeit y da aun un paso más en dirección al idealismo lingüístico, pues el modelo textual actúa "mediatizando" toda comprensión de sí y del mundo. ${ }^{14}$ Romano entiende que Ricœur, kantianamente, les da a las categorías del texto una "función constituyente para la experiencia misma" (Romano, 2010, p. 883). La tesis de Temps et récit es precisamente: "no hay experiencia humana que no esté ya mediada por sistemas simbólicos y, entre ellos, por narraciones" (Ricœur, 1983, p. 113). Romano concluye preguntándose si esta "hipóstasis del texto" y la presuposición del "carácter amorfo de la experiencia" no conllevan la imposibilidad de articular de modo adecuado fenomenología y hermenéutica (cf. Romano, 2010, p. 884).

Según Romano, el carácter extremo de esta propuesta se observa también en el hecho de que, a diferencia de Gadamer que distingue entre la comprensión espontánea y la interpretación de textos, para Ricœur comprender e interpretar son coextensivos. De este modo, se puede llegar a una "consecuencia desastrosa": "Si la interpretación comienza en el nivel más elemental de la comprensión, si la interpretación es una actividad que se expresa en signos, toda interpretación debe reenviar, a su vez, a una nueva interpretación y el regreso al infinito es inevitable. Nada puede ser jamás comprendido y, por tanto, nada puede jamás ser interpretado tampoco: la distinción comprender/interpretar es destruida en su principio mismo" (Romano, 2010, p. 885). Romano va a insistir en la importancia de la distinción entre comprensión e interpretación como un modo de asegurar que se cumpla la "exigencia fenomenológica" en la articulación con la hermenéutica.

Ahora bien, esta lectura de Gadamer y Ricœur, como el propio Romano advierte, puede ser objetada. Es posible conciliar la crítica al "idealismo lingüístico" con la afirmación de la Sprachlichkeit. En rigor, la frase de Gadamer debe ser entendida, simplemente, como la afirmación de que es en el lenguaje en donde experimentamos el ser. Pero el hecho de que necesitemos del lenguaje para pensar lo que nos es dado previamente en la experiencia, no implica que se pueda afirmar que nada nos es dado fuera del lenguaje.

14 “Comprenderse es comprenderse ante el texto" (Ricœur, 1986, p. 33). 
Conviene detenerse brevemente en algunos rasgos de la concepción gadameriana del lenguaje. Gadamer entiende que no debe pensarse al lenguaje como una mera herramienta para el pensamiento. Este es -según Gadamer- el problema del "olvido del lenguaje" (Sprachvergessenheit) en el pensamiento occidental: se cree que el pensamiento se relaciona directamente con el mundo, que el lenguaje es un simple instrumento del que se vale el pensamiento (cf. 1999, p. 422). Esta concepción, como bien explica Grondin, conlleva un nominalismo en sentido amplio, pues se entiende que la palabra es una designación o un signo que se agrega con posterioridad a la cosa o al pensamiento (cf. Grondin, 2003, p. 201). Por el contrario, Gadamer sostiene que no existe tal distancia con relación al lenguaje, sino más bien una pertenencia respecto de él: nuestro ser-en-el-mundo y nuestra comprensión son lingüísticos, existe un vínculo fundamental entre el lenguaje y el mundo. Esto implica que, por un lado, el mundo solo es mundo en tanto es expresado en el lenguaje, pero también, por otro lado, el lenguaje es tal en tanto el mundo se representa en él: “...el lenguaje no afirma una existencia autónoma frente al mundo que habla a través de él" (Gadamer, 1999, p. 447).

Pero, además, el ser solo puede presentarse en el lenguaje, pero esto no implica que el ser quede circunscripto de modo definitivo al lenguaje. Por eso Gadamer aclara que esta "lingüisticidad de la comprensión no quiere decir que toda experiencia del mundo deba cumplirse bajo el modo del lenguaje o en el lenguaje" (Gadamer, 1996, p. 46). Es más, existe una carencia esencial en el lenguaje respecto del ser: el campo de lo decible (el ser) excede ampliamente el campo de lo que efectivamente puede decirse. Gadamer insiste en el carácter finito de nuestros enunciados lingüísticos, que nunca agotan lo que puede decirse respecto de algo y que entran en tensión con un "querer decir" infinito. "Todo hablar humano es finito, en el sentido de que en el yace la infinidad de un sentido por desplegar e interpretar [auslegen]" (Gadamer, 1999, p. 462). El lenguaje se articula desde una tensión entre su capacidad de decir la experiencia, de dar cuenta de su intimidad y familiaridad, y nuestra finitud que lo torna siempre insuficiente. La comprensión se caracteriza por la búsqueda de la palabra adecuada, que está animada por ese "querer decir" infinito, siempre en tensión con las limitaciones del decir efectivo. ${ }^{15}$

Finalmente, Gadamer destaca que nuestra pertenencia al lenguaje debe ser estudiada, no desde el signo (que implicaría introducir una distancia objetivante), sino desde la vida misma del lenguaje en el diálogo. Todo enunciado adquiere sentido a partir de la estructura dialógica de la pregunta y la respuesta, de la que no podemos tomar distancia, pues siempre nos encuentra ya implicados.

Respecto de Ricœur también puede formularse algunas aclaraciones pertinentes. En su artículo "Langage" se lee: "El lenguaje aparecería entonces no solamente como un mediador entre el hombre y el mundo, sino más precisamente como un intermediario [échangeur] entre dos exigencias, una exigencia de logicidad que le da un télos, y una exigencia de fundamento en lo antepredicativo que le da un arché. La función simbólica se comprende por esta doble exigencia" (Ricœur, 1971, p. 776). Son estos dos aspectos, el télos y el arché, los que son desplegados en el círculo entre

15 Esto es lo que Gadamer llama la "estructura especulativa del lenguaje”: “...el lenguaje tiene algo de especulativo en un sentido completamente distinto [...] como realización de sentido, como acontecer del habla, del entenderse, del comprender. Esta realización es especulativa en cuanto que las posibilidades finitas de la palabra están asignadas al sentido de su referencia como a una orientación hacia el infinito. El que quiere decir algo busca y encuentra las palabras con las que hacerse comprensible al otro" (Gadamer, 1999, pp. 472-473). 
epistemología (télos) y ontología (arché) en el desarrollo de la "vía larga".

Pero, además, la "exigencia fenomenológica", es decir, "la exigencia de fundamento en lo antepredicativo" queda claramente enunciada en su artículo "Phénoménologie et Herméneutique. En venant de Husserl". Allí la tesis hermenéutica de la Sprachlichkeit es conciliada con la afirmación del carácter derivado de las significaciones lingüísticas con relación a la experiencia: "Si bien es cierto que toda experiencia tiene una 'dimensión lingüística', y que esta Sprachlichkeit impregna y atraviesa toda experiencia, sin embargo, una filosofía hermenéutica no debe comenzar por la Sprachlichkeit. En primer lugar debe decir lo que llega al lenguaje" (Ricœur, 1986, p. 59). La hermenéutica reconoce una raíz fenomenológica y es, precisamente, este reenviar del orden lingüístico a la estructura de la experiencia el "presupuesto fenomenológico más importante de la hermenéutica” (Ricœur, 1986, p. 60).

\section{Las tensiones entre fenomenología y hermenéutica en Sein und Zeit}

A continuación de sus análisis sobre Gadamer y Ricœur, Romano sostiene que las tensiones entre fenomenología y hermenéutica en estos autores se explican, precisamente, porque los dos buscan hacer lugar a ambas exigencias (la fenomenológica y la hermenéutica). Romano destaca que las exigencias no son incompatibles, pero pueden volverse contradictorias si se sostiene, de modo extremo: 1) que toda experiencia es lingüística en su esencia (estructurada por sistemas conceptuales) y 2) que toda experiencia es ya una interpretación.

Las inconsistencias en Gadamer y Ricœur puede explicarse a partir de Heidegger, pues ya se encuentran presentes en su obra. Si bien en Sein und Zeit, Heidegger hace lugar a la exigencia fenomenológica de un sentido prelingüístico que precede toda enunciación, Romano destaca que la Seinsfrage es formulada de modo ambiguo. Heidegger no distingue la pregunta por el sentido del ser de la pregunta por el sentido del "ser" como verbo comprendido en toda predicación. ${ }^{16}$ Esto nos enfrenta al siguiente dilema: 1) o se mantiene la exigencia fenomenológica y se disocia la pregunta por el ser de la pregunta por el "ser", 2) o se admiten que las dos preguntas son inseparables y se hace depender de una capacidad lingüística todo descubrimiento intramundano del ente (cf. Romano, 2010, pp. 889-890).

Pero, además, al subordinar toda manifestación de fenómenos a una comprensión del ser, es decir, al identificar fenomenología y ontología, Heidegger -según Romano- intelectualiza la percepción. Heidegger afirma que "toda percepción de un útil a la mano es comprensora-explicitante [verstehend-auslegend]", ${ }^{17}$ pero entonces,

16 Romano cita el siguiente pasaje: “Todo el mundo comprende esto: 'el cielo es azul', 'yo soy una persona de buen humor', etc. Pero esta comprensibilidad 'de término medio' no hace más que mostrar la incomprensibilidad. [...] El hecho de que vivamos en cada caso en cierta comprensión del ser, y que a la par el sentido del ser sea esbozado en la oscuridad, prueba la fundamental necesidad de reiterar la pregunta que interroga por el sentido del "ser"” (GA 2, p. 6).

17 El pasaje completo dice: "Pero si ya toda percepción de un útil a la mano es comprensora-explicitante y deja comparecer circunspectivamente algo en cuanto algo, ¿no quiere entonces decir esto que primero se experimenta una cosa que está puramente ahí, y que luego se la entiende en cuanto puerta o en cuanto casa? Esto sería malentender la específica función que en el "abrir" tiene la explicitación. La explicitación no arroja cierto "significado" sobre el nudo ente que está-ahí, ni lo reviste con un valor, sino que lo que comparece dentro del mundo, ya tiene siempre, en cuanto tal, una condición respectiva abierta en la comprensión del mundo, y esta condición queda expuesta por medio de la explicitación" (GA 2, p. 199). Cabe destacar que el hecho de que no 
se pregunta Romano, ¿cómo mantener la distinción entre percibir e interpretar (o explicitar)? ¿Cómo mantener el concepto de interpretación (o explicitación) si éste ya no trata sobre un interpretatum que la precede? (cf. 2010, pp. 890-891). Con la Kehre, según Romano, la tensión se resuelve porque Heidegger opta por un idealismo lingüístico: "el lenguaje es la casa del ser" (GA 5, p. 310).

Finalmente, a estas tensiones en Sein und Zeit, se agrega también otra dificultad. En el $\S 7$ se encuentran dos afirmaciones que parecen contradecirse entre sí. Por un lado, Heidegger afirma que la descripción de los fenómenos es hermenéutica: "El sentido metodológico de la descripción fenomenológica es Auslegung" (GA 2, p. 50). Pero por otro lado, al reparar en el fenómeno eminente del ser, Heidegger sostiene-según Romano- que la donación misma de los fenómenos es hermenéutica. La aparición del fenómeno del ser del Dasein requiere una tarea hermenéutico-destructiva. Romano concluye pues que Heidegger está sosteniendo que la Auslegung no opera sobre fenómenos dados previamente, no nos hace "ver mejor" a los fenómenos, sino que simplemente nos posibilita verlos por primera vez. "Si todo lo que es fenómeno no es solamente descripto, sino también dado por medio de una interpretación, nuevamente, no hay más ni fenómenos ni interpretación" (Romano, 2010, pp. 893894).

Asimismo, Romano destaca que la afirmación heideggeriana que sostiene que el acceso a los fenómenos es sólo posible por la interpretación, contradice el concepto heideggeriano de fenómeno. Si el fenómeno solo se da por intermedio de la interpretación, se abren dos posibilidades igualmente inaceptables: 1) o se transforma a la "cosa misma" en un polo teleológico similar a la idea kantiana, ${ }^{18} 2$ ) o se cae en un perspectivismo extremo en el sentido nietzscheano.

Romano plantea cuatro preguntas para aclarar su posición: 1) ¿La experiencia incluye una comprensión? 2) ¿La experiencia incluye una interpretación? 3) ¿La descripción de la experiencia incluye una comprensión? 4) ¿La descripción de la experiencia incluye una interpretación? Las respuestas correctas, según Romano, son las siguientes: 1) Sí, la experiencia incluye una comprensión. A través de nuestras posibilidades prácticas, nace una comprensión del mundo con un sentido prelingüístico que no está todavía articulado como un pensamiento en sentido estricto. 2) Depende, no siempre la experiencia incluye una interpretación. Se puede comprender sin interpretar, pero no podemos interpretar sin comprender. Es necesaria una interpretación si la comprensión es deficiente, es decir, si se produce una ruptura con la comprensión espontánea propia de nuestra cotidianeidad con el mundo y con los otros. 3) Sí, la descripción de la experiencia incluye una comprensión, pues describir es ciertamente una actividad lingüística. Allí se pone en juego tanto una comprensión de lo que describo como una comprensión de las palabras que utilizo para describir. 4) No siempre la descripción de la experiencia incluye una

sea posible una distinción cronológica no implica que no haya una anterioridad de la comprensión respecto de la explicitación, como aquello en el que esta última se funda y deviene posible.

18 Romano señala que, en algún sentido, Heidegger cayó en esta solución en el curso de invierno de 1925/1926: “El título ‘fenómeno' significa siempre en cierto modo una tarea [Aufgabe]: en sentido negativo, el asegurarse frente a opiniones preconcebidas y prejuicios; en sentido positivo, una tarea en el sentido de que el análisis de los llamados fenómenos tiene que aclararse a sí mismo qué opiniones preconcebidas carga sobre los objetos de la filosofía, pues finalmente puede mostrarse que no cabe la ausencia de estas opiniones preconcebidas y que, por consiguiente, la crítica de las opiniones preconcebidas esenciales representa una parte esencial de la investigación filosófica" (GA 21, p. 33). 
interpretación: eso depende del tipo de descripción. Si la frase "el cielo es azul" es ya una descripción, entonces allí no interviene una interpretación de la percepción del cielo. Pero la fenomenología se interesa por descripciones más complejas, que incluyen elementos teóricos determinados por la historia de la filosofía. En estos casos, sostiene Romano, la hermenéutica es un correctivo contra el dogmatismo de la intuición de esencias (cf. 2010, pp. 896-898).

Estas afirmaciones de Romano no hacen más que explicitar las ideas del propio Heidegger: en el nivel antepredicativo de la experiencia contamos con una comprensión que ya es explicitación desde un "en tanto que" hermenéuticoexistencial, pero que puede no requerir una explicitación en términos de un "en tanto que" apofántico. Y, en todo caso, el "en tanto que" apofántico de la enunciación debe fundarse siempre en el "en tanto que" hermenéutico-existencial.

Por otra parte, si bien es cierto que existen estas tensiones, conviene destacar que Heidegger se inclina por la "exigencia fenomenológica", pues sostiene en Sein und Zeit $\mathrm{y}$, en general en esos años, una primacía de la experiencia antepredicativa frente a la instancia predicativa y una anterioridad de la comprensión frente a toda tarea de explicitación, interpretación y enunciación. Esto se observa en toda su radicalidad con la tesis de la anterioridad de la nada respecto del no y la negación en „Was ist Metaphysik?”: "nosotros afirmamos: la nada [das Nichts] es más originaria que el no [das Nicht] y la negación [die Verneinung]" (GA 9, p. 108). Heidegger allí enfatiza que la imposibilidad formal de una enunciación adecuada en el plano lógico no invalida la pregunta por la nada, pues tenemos una experiencia de ella. La nada se da en una experiencia fundamental (la angustia). Este plano ontológico (o meontológico) es fundante respecto del plano lógico. El "nadear" o "desistir" (Nichten) de la nada se manifiesta en diversos comportamientos del Dasein y no puede ser reducido a la "negación” (Verneinung) lógica (cf. GA 9, pp. 116-117).

\section{Reglas de la experiencia y verdades de esencia}

Es necesario enfatizar, según Romano, que: "La hermenéutica fenomenológica sólo puede formularse de manera coherente si se admite el nivel pre-hermenéutico de una comprensión espontánea operando en la experiencia misma, una experiencia perceptiva no mediada por signos. [...] De no ser así ¿qué significaría exactamente la referencia a la "cosa misma" a la que la fenomenología tiene por tarea reconducirnos?" (2010, p. 895). Y es precisamente este nivel pre-hermenéutico el que puede imponer reglas. Si bien la hermenéutica no puede responder a normas o criterios exactos que se apliquen mecánicamente, sí debe seguir las "reglas de la experiencia" (cf. 2010, p. 901). Estas "leyes" no son unívocas ni permiten una aplicación unívoca a todos los casos, sino que -como la phrónesis aristotélica- se ajustan a cada caso. Se trata de reglas que apelan a la experiencia para ser correctamente aplicadas y que, en su aplicación, demandan la deliberación y la reflexión. Seguir estas reglas exige recurrir al juicio, tener una sensibilidad para el contexto y una capacidad de discernimiento de lo particular (cf. Romano, 2015, pp. 475-477). Romano propone la tarea de la traducción como ejemplo para entender el modo en que operan estas reglas: "No hay, en sentido estricto, un método de traducción, si por eso entendemos un sistema de equivalencias (como el que podría aplicar un programa de computación) en el que bastaría basarse en todos los casos, solo hay reglas / métodos falibles y dotados de 
un alcance limitado, pues son tributarios de la particularidad del contexto, y exigen una experiencia para poder ser aplicados juiciosamente" (2015, pp. 477-478). Estas "reglas de la experiencia" constituyen la particularidad del método hermenéutico, que no tiene nada de arbitrario, aunque no responda a normas exactas.

Asimismo, Romano destaca que si la hermenéutica -en tanto doctrina filosóficaaspira a la verdad, ésta debe basarse en verdades que no se encuentran subordinadas a las condiciones históricas y finitas de la interpretación. Romano entiende que "una hermenéutica auténtica es fenomenológica" en tanto enraíza sus descripciones en verdades de esencia pre-hermenéuticas (cf. 2010, pp. 901-902). ${ }^{19}$ Hablar de esencias no implica tener que comprometerse con ideas situadas en un dominio suprasensible, ni de carácter eterno e inmutable. Hablar de esencias no conlleva necesariamente suscribir a un realismo de los universales. En contra de estas equiparaciones, Romano postula una concepción adverbial de la esencia que se basa en el siguiente principio: "Las esencias no son ni objetos situados en un cielo inteligible, ni objetos localizables aquí abajo; no son de ningún modo objetos. En otros términos, no hay esencias a título de objetos ideales, pero las cosas son esencialmente tales o tales" (2016, p. 200).

Romano entiende necesario sostener una doctrina de las esencias, principalmente, para no caer en la reducción del convencionalismo que, en una suerte de neonominalismo, las reconduce al lenguaje. Romano afirma que este convencionalismo es insostenible, pues confunde las necesidades de las cosas con las necesidades de los conceptos. El convencionalismo entiende que solo existe una necesidad: la necesidad establecida convencionalmente, las necesidades del lenguaje. De este modo, según Romano, se desconocen las necesidades reales. Para dar cuenta de este problema, ofrece un argumento a partir de la comparación de dos afirmaciones: 1) "Ningún soltero está casado" y 2) "Ningún objeto uniformemente rojo es uniformemente verde". La primera proposición afirma una necesidad conceptual, la segunda una necesidad real. La verdad de la segunda proposición no trata sobre la aplicación correcta de la palabra "rojo", sino que afirma que un objeto rojo no puede ser a la vez verde. Se trata de una verdad de esencia propia del dominio de los colores.

Ahora bien, esta afirmación de una doctrina de las esencias en fenomenología debe tomar distancia respecto de la concepción husserliana de las esencias y de su acceso a ellas. Según Romano, la eidética debe ser rechazada por dogmática. No es posible algo así como una intuición de la esencia. Romano sostiene que hay que criticar el platonismo de Husserl. Si efectivamente los fenómenos prescriben su propio modo de abordaje, "la hermenéutica cumple a la fenomenología", la hace alcanzar su cometido, pues corrige la desviación cartesiana de la fenomenología husserliana basada en la intuición de ideas ahistoricas.

Asimismo, "la hermenéutica cumple a la fenomenología" porque la hace abandonar la pretensión de una Vorurteilslosigkeit. No hay descripciones puras, no hay descripciones que se puedan sustraer de sus presupuestos históricos. Y, en este sentido, "las descripciones de esencias constituyen la primera palabra, pero de ninguna manera la última palabra de la tarea fenomenológica" (Romano, 2016, p. 205). Ciertamente, la fenomenología no debe olvidar sus condiciones históricas concretas, es decir, la finitud de la razón, pero esto no implica que no puede sostenerse una doctrina de las esencias.

19 Romano dedica tres capítulos de Au cæur de la raison... (X, XI y XII) a esclarecer su noción de esencia (cf. pp. 373-466). 
Por otra parte, Romano destaca que las "verdades de esencia" que él afirma no se sustraen a la posibilidad de revisión en el marco de la discusión argumentada (cf. 2016, p. 206). Por este motivo, sobre el final del capítulo dedicado al vínculo entre fenomenología y hermenéutica, se afirma que la descripción de la comprensióninterpretación es revisable, como toda descripción, pero que no todo es revisable en la descripción. Si los argumentos que dan cuenta de las verdades de esencia iniciales no se revelan insuficientes o superables por una mejor argumentación, entonces no todo es idealmente revisable sin más (cf. Romano, 2010, pp. 903-904).

\section{La fenomenología hermenéutica marioniana}

¿Qué ocurre en el caso de Marion? ¿Existe la tensión entre fenomenología y hermenéutica? Podría esperarse que el heideggerianismo de Marion lo llevara a caer en la misma indecisión entre la "exigencia fenomenológica" y la "exigencia hermenéutica". Sin embargo, si se presta atención, en primer lugar, a la distinción entre donación y fenomenalización, y, en segundo lugar, a las afirmaciones de Reprise du donné, puede advertirse que, efectivamente, Marion comparte las tesis de Romano respecto de la articulación entre fenomenología y hermenéutica.

La diferencia entre la instancia de donación y la instancia de fenomenalización que puede no ser una diferencia cronológica, pero que siempre es lógica o, mejor aún fenomenológica- permite advertir que no es lo mismo decir que la donación misma de los fenómenos es hermenéutica, que decir que la hermenéutica hace aparecer a los fenómenos. Esta crítica que Romano plantea respecto de Heidegger no sería aplicable a Marion. Ahora bien, puede parecer que la ausencia de distancia cronológica entre la llamada y la respuesta lleva a la hermenéutica marioniana a caer en un "idealismo lingüístico". Sin embargo, cabe señalar que la fenomenología de la donación se presenta como una crítica de todo tipo de idealismo: 1) es crítica del "idealismo trascendental" (el adonado no cumple una función de sujeto constituyente, sino de testigo de la donación), 2) pero también es crítica del "idealismo lingüístico". La hermenéutica marioniana debe dar al fenómeno su sentido, el sentido que el fenómeno se da a sí mismo, el sentido que se plasma en el darse antepredicativo, en la contraexperiencia. ${ }^{20}$ Ninguna convención lingüística o cultural puede imponer de modo externo, "desde afuera", un sentido al acontecer del fenómeno. El fenómeno se da su propio sentido, esa es la verdad esencial que puede captarse en la contra-experiencia. Marion es crítico de la noción "ontoteológica" de esencia que puede asociarse a la concepción aristotélica de ousía (cf. 1998, pp. 213-224 y 2012, p. 78), pero acepta una "verdad paradojal" 21 que demanda una exposición en primera persona a lo que se da en sus propios términos. ${ }^{22}$ Para acceder a la verdad del fenómeno saturado, para

20 "La hermenéutica no da un sentido a lo dado, fijándolo y decidiéndolo, sino que, cada vez, da su sentido, es decir, da lo que hace aparecer a esto dado como él mismo, como un fenómeno que se muestra en sí y para sí” (Marion, 2016, p. 40).

21 Marion llama "paradojas" a los fenómenos saturados. "Paradoja significa lo que va en contra (pára-) de la opinión corriente, de la apariencia, siguiendo los dos sentidos obvios de la dóxa; pero también significa lo que va en contra de la expectativa - '[...] praeter expectationem offertur'-, lo que acaece contra toda expectativa de representación, de la intención, en resumen, del concepto. La paradoja pertenece sin duda al dominio de la verdad, salvo por una característica: su donación contraviene, en su intuición, lo que la experiencia anterior debería permitir prever razonablemente" (Marion, 1998, p. 315).

22 "Pues la verdad no se des-cubre jamás inmediatamente como un espectáculo ni se pronuncia inmediatamente como un enunciado; ella sólo se des-cubre mediatamente, afrontando, atravesando y colmando la distancia 
acceder a $s u$ sentido hay que aceptar el riesgo de abandonar la lógica del espíritu y recibirlo desde la lógica del corazón. Se requiere una "hermenéutica del amor"23 capaz de desplazar la concepción de la ousia o "esencia" como algo que se puede poseer hacia una noción de ousia o "esencia" como don. ${ }^{24}$

Pero ¿cómo se explicita ese sentido que el fenómeno se da a sí mismo? ¿Hay una Sprachlichkeit de la contra-experiencia? Ciertamente este es un aspecto de la hermenéutica marioniana que aún no ha sido desarrollado. En este sentido, considero que la propuesta de Javier Bassas Vila (2009) es muy valiosa para señalar un posible camino hacia un "lenguaje saturado" bajo la modalidad dionisíaca del "como" de la alabanza. Pero, además, puede indagarse en la estructura de la llamada y la respuesta en búsqueda de una concepción del lenguaje, estructura que es traducida por Marion en términos hermenéuticos como la de la pregunta y la respuesta. Gadamer sostiene que es en el diálogo en donde se encuentra el sentido del lenguaje. Cuando se detiene en la alabanza, Marion cita a Lévinas: "La esencia del discurso es la oración" (Lévinas, 2014, p. 19). La oración es la esencia del discurso porque el lenguaje, en su origen, refiere a una relación que se da en términos antepredicativos. La manifestación del Otro, el rostro, es lenguaje sin palabras, es el lenguaje originario que se da en el modo de la llamada. Según Lévinas, la "relación metafísica", la relación entre lo Mismo y lo Otro es el lenguaje, pues el lenguaje establece una relación en la que el Otro no pierde su transcendencia (cf. 1963, pp. 28-29). Esta "relación metafísica" "está antes de la proposición negativa o afirmativa, instaura el lenguaje en el que ni el 'no' ni el 'sí' son la primera palabra" (p. 32).

La primera palabra que instaura esta relación, que es lenguaje, es la llamada, la interpelación, el mandamiento que se manifiesta en el rostro del Otro. Esta es la "esencia ética" del lenguaje que se identifica con el rostro como expresión. Dice Lévinas: "Este vínculo entre la expresión y la responsabilidad, esta condición o esta esencia ética del lenguaje, esta función del lenguaje, anterior a todo develamiento del ser y a su frío esplendor, permite sustraer el lenguaje a su sujeción respecto de un pensamiento preexistente, a propósito del cual el lenguaje no tendría sino la función servil de traducir afuera o de universalizar sus movimientos anteriores" (1963, p. 219).

El núcleo esencial del lenguaje es oración porque es relación con el Otro. El sentido surge del rostro del Otro. El rostro aporta la primera significación. En un sentido ciertamente diferente, puede afirmarse que Totalité et infini responde a la "exigencia hermenéutica" que sostiene que "el lenguaje es constitutivo del pensamiento en sentido fuerte" (Romano, 2010, p. 887): "El discurso condiciona el pensamiento, porque el primer inteligible no es un concepto, sino una inteligencia, cuyo rostro enuncia la exterioridad inviolable profiriendo el 'no matarás'. La esencia del discurso es ética. Al enunciar esta tesis, se rechaza el idealismo" (Lévinas, 1963, p. 238).

[écart] entre lo que se da y lo que eventualmente se muestra" (Marion, 2014, p. 25).

23 Marion destaca que la verdad exige el amor: "Así, la metafísica se descubre destituida de la verdad, no porque una instancia más elevada destituiría la verdad [...], sino porque la verdad exige, más que el método, el ejercicio de la caridad en respuesta al amor propio que la tiraniza [...] La metafísica pretende acceder a la verdad solo por el método, como si se tratara de un objeto a construir; ella engaña y se engaña ya que es necesario amar la verdad para acceder a ella" (Marion, 1986, p. 363).

24 Este es el sentido de la lectura de la parábola del hijo pródigo que Marion presenta en Dieu sans l'être: "La ousia se inscribe en el juego del don, del abandono y del perdón, que hace de ella la moneda de un intercambio totalmente diferente al de los entes" (Marion, 1982, p. 146). 
La concepción lévinasiana del lenguaje permite pensar una hermenéutica que no se introduce como una mediación y que no cae en ningún "idealismo lingüístico" porque se basa en una experiencia previa, la de la relación metafísica, que, además, tiene el carácter de un lenguaje antepredicativo, un lenguaje pragmático. El sentido antepredicativo fenomenológico que funda el sentido lingüístico se manifiesta en la estructura misma del lenguaje, en la relación metafísica entre lo Mismo y lo Otro. Cabe preguntarse, pues, si Marion no asume esta concepción lévinasiana cuando afirma que "se trata de pasar de la predicación al dirigirse a..." (2012, p. 279).

Teniendo en cuenta estos lineamiento que se encuentran latentes en la obra marioniana como posibilidades a desplegar, puede afirmarse que la fenomenología de la donación es una fenomenología hermenéutica de la donación que acepta la fórmula de Romano en todo su alcance: "la hermenéutica auténtica es una fenomenología y la fenomenología solo se cumple como hermenéutica": 1) Es una hermenéutica que asume la primacía de la fenomenología en tanto acepta la anterioridad de la instancia antepredicativa y extrae su sentido de ella. 2) Es una fenomenología que se cumple como hermenéutica porque la fenomenología de la donación no acepta ninguno de los "dogmatismos" husserlianos: el sujeto constituyente es sustituido por el adonado, y la búsqueda de un comienzo absoluto libre de supuestos es abandonada, pues toda búsqueda de la verdad a partir de una pre-visión es sustituida por una post-visión que espera a la manifestación del fenómeno y se involucra en ella asumiendo las limitaciones de la finitud y de la Geworfenheit.

\section{Referencias bibliográficas}

Bassas Vila, J.: "Écriture phénoménologique et théologique: Fonctions du 'comme', 'comme si' et 'en tant que' chez Jean-Luc Marion”, Studia Phcenomenologica, special issue, pp. 135-155, 2009.

Gadamer, H.-G.: La philosophie herméneutique, trad. J. Grondin, Paris, PUF, 1996.

Gadamer, H.-G.: Gesammelte Werke. Band 1: Hermeneutik I. Wahrheit und Methode, Tübingen, Mohr Siebeck, 1999.

Greisch, J.: "L'herméneutique dans la 'phénoménologie comme telle': trois questions à propos de 'Réduction et donation", Revue de métaphysique et de morale, 96, pp. 43-63, 1991.

Grondin, J.: "La phénoménologie sans herméneutique: Jean-Luc Marion, Réduction et donation", Internationale Zeitschrift für Philosophie, 1, pp. 146-153, 1992.

Grondin, J.: Introducción a Gadamer, trad. C. Ruiz-Garrido, Barcelona, Herder, 2003.

Lévinas, E.: Totalité et Infini. Essai sur l'extériorité, La Haye, Martinus Nihjoff, 1963.

Lévinas, E.: “L'ontologie, est-elle fondamentale ?", en: Entre nous. Essais sur le penser-àl'autre. Paris, LGF, 2014.

MacKinlay, Sh.: Interpreting Excess. Jean-Luc Marion, saturated phenomena, and hermeneutics, New York, Fordham University Press, 2010.

Marion, J.-L.: Dieu sans l'être, Paris, Fayard, 1982.

Marion, J.-L.: Sur le prisme métaphysique de Descartes. Constitution et limites de l'ontothéo-logie cartésienne, Paris, PUF, 1986.

Marion, J.-L.: Étant donné. Essai d'une phénoménologie de la donation, seconde édition corrigé, Paris, PUF, 1998.

Marion, J.-L.: Le croire pour le voir, Paris, Communio Parole et silence, 2010. 
Marion, J.-L.: La rigueur des choses. Entretiens avec Dan Arbib, Paris, Flammarion, 2012.

Marion, J.-L.: "Quelques précisions sur la réduction, le donné, l'herméneutique et la donation”, en Sommer, C. (éd.), Nouvelles phénoménologies en France. Actes des journées d'étude autour de Hans-Dieter Gondek et László Tengelyi Neue Phänomenologie in Frankreich, Paris, Hermann, pp. 215-234, 2014a.

Marion, J.-L.: "Une question de réponse", en Ph. Capelle-Dumont et Y. Courtel (éds), Religion et liberté, Strasbourg, Presses Universitaires de Strasbourg, 2014b.

Marion, J.-L.: Reprise du donné, Paris, PUF, 2016.

Ricœur, P.: "Langage (Philosophie)", en AA. VV., Encyclopcedia universalis, tome 9, Paris, Encyclopædia Universalis SA, 1971.

Ricœur, P.: Temps et récit I, Paris, Seuil, 1983.

Ricœur, P.: Du texte à l'action. Essais d'herméneutique II, Paris, Seuil, 1986.

Ricœur, P.: Le conflit des interprétations. Essais d'herméneutique I, Paris, Seuil, 2013.

Roggero, J. L., "Los cuatro niveles de hermenéutica en la fenomenología de J.-L. Marion", Revista de Filosofía. Universidad Complutense de Madrid, vol. 45, núm. 1, pp. 141-160, 2020.

Romano, C.: Au cœur de la raison, la phénoménologie, Paris, Gallimard, 2010.

Romano, C.: “La règle souple de l'herméneute", Critique, 6, 817-818, pp. 464-479, 2015.

Romano, C.: “Avons-nous besoin des essences en philosophie?”, en Ph. Cabestan (éd.), L'événement et la raison. Autour de Claude Romano, Paris, Le Cercle Herméneutique, 2016.

Serban, C.: "La méthode phénoménologique, entre réduction et herméneutique", Les études philosophiques, 1, 100, pp. 81-100, 2012.

Vallée, M.-A.: Gadamer et Ricour. La conception herméneutique du langage, Rennes, Presses Universitaires de Rennes, 2012. 